Itinéraires Itinéraires

Littérature, textes, cultures

2010-3 | 2010

Médiévalisme

\title{
Le médiévalisme entre hypnose numérique et conservatisme rétro
}

Anne Larue

\section{OpenEdition}

\section{Journals}

Édition électronique

URL : http://journals.openedition.org/itineraires/1830

DOI : 10.4000/itineraires. 1830

ISSN : 2427-920X

Éditeur

Pléiade

\section{Édition imprimée}

Date de publication : 1 novembre 2010

Pagination : 87-96

ISBN : 978-2-296-13150-7

ISSN : 2100-1340

\section{Référence électronique}

Anne Larue, « Le médiévalisme entre hypnose numérique et conservatisme rétro », Itinéraires [En ligne] 2010-3 | 2010, mis en ligne le 01 novembre 2010, consulté le 01 mai 2019. URL : http://

journals.openedition.org/itineraires/1830; DOI : 10.4000/itineraires.1830

\section{(ब) $(\Theta \Theta$}

Itinéraires est mis à disposition selon les termes de la licence Creative Commons Attribution - Pas d'Utilisation Commerciale - Pas de Modification 4.0 International. 


\title{
Le médiévalisme entre hypnose numérique et conservatisme rétro
}

\begin{abstract}
This paper examines the importance of medievalism in the resistance against backlash. Medievalism prevents fantasy in spite of its conservative touch of " retrolution » from becoming a New Age ineffective phenomenon. It links the blossoming of new popular fiction with an ideological and political aim. Medfan on the Internet is not at all a critical frame; but fantasy books, carried by the medfan movement, sometimes become modern spinozist theological and political treatises available in supermarkets.
\end{abstract}

Keywords : medfan, fantasy, backlash, ideology, politics

Mots clés : medfan, fantasy, backlash, idéologie, politique

Le « retour au médiéval » qu'on observe aujourd'hui dans la littérature de l'imaginaire et les médias culturels numériques est une manière parmi d'autres de résister au désenchantement du backlash. Selon Susan Faludi, le backlash est un retour en arrière ${ }^{1}$, une « revanche » du système patriacal par rapport à l'époque de la contre-culture. François Cusset parle à ce sujet de « cauchemar des années 1980 ». Malgré l' « exception française » - et la venue au pouvoir des socialistes - l'idéologie mondiale se durcit alors globalement. Rappelons-nous : golden boys, game boys, adulation des "créateurs » de publicité, valorisation mondiale de «l'entreprise » et du « libéralisme » en Occident, montée des violences religieuses, tout ce que dénonce François Cusset marque une violente réaction contre les idées de l'époque précédente ${ }^{2}$. La «fin des grands récits », développée

1. Susan Faludi, Backlash. The Undeclared War Against Women, New York, Crown Publishers, 1991.

2. François Cusset, La Décennie. Le grand cauchemar des années 1980, Paris, La Découverte, 2006. 
par Lyotard ${ }^{3}$, est détournée et récupérée pour faire croire que, désormais, il n'existe plus d'idéologie. Pourtant, l'installation progressive de la « pensée » unique du marché - vraiment un «grand récit » cohérent et triomphant, aujourd'hui - contredit ipso facto cette façon de voir. Nous baignons aujourd'hui dans la violence « passive agressive », comme disent les Anglo-Saxons : contre cette violence sans nom officiel, une violence qui ne dit que rarement son nom, chacun rentre chez soi et se logue en tant que hobbit - pour le dire vite. Face à cet envahissement d'un système du monde qu'on pourrait dire « de la Patriarquie Unie », avec montée de l'impérialisme marchand à la fin de la Guerre froide, effondrement du bloc communiste, mondialisation du matraquage publicitaire, «barbarie douce $^{4} \gg$, selon les termes de Jean-Pierre Le Goff, et un mépris des femmes que n'auraient pas désavoué les futuristes fascistes comme Marinetti au début du siècle, le médiévalisme entreprend une sourde lutte sur le terrain de l'imaginaire, investi dès lors d'une puissance de contestation.

Se réfugier derrière son ordinateur pour retrouver le Moyen Âge est-il la marque d'un refus de lutter politiquement, d'un découragement, d'un repli sur des valeurs individualistes qui se trouvent elles aussi mises en exergue par les années 1980, comme si le poison générait son antidote, la violence-même les moyens de la fuir? Le médiévalisme apparaît en effet au premier abord comme un refuge, un " escapisme », une manière de se protéger dans son château-fort immuable et imprenable. Cette attitude relèverait d'un certain conservatisme nostalgique, rassuré par une société très hiérarchisée qui aurait duré mille ans, soi-disant sans changements notables : c'est ce que j'ai appelé « l'effet Malevil », en référence au roman de Robert Merle ${ }^{5}$. Le médiévalisme comble un tel fantasme de stabilité. Justement, dans le monde du jeu vidéo, on ne transforme pas le monde dans lequel on agit. Ce n'est pas un hasard dès lors que de nombreux jeux vidéo voient perpétuer racisme, sexisme, mondialisation, capitalisme et stéréotypes, le jeu étant de surcroît soumis à l'invariant impensé du meurtre : la chose à faire est de tuer (des monstres, des opposants), cela va de soi, personne n'imagine une autre façon de faire. Plus que jamais, un travail foucaldien de critique des discours dominants d'une société demande à être mené dans le cadre d'une analyse des jeux médiévaux, qui profitent d'un retour fantasmé au passé pour conforter les invariants culturels de l'espace social.

3. Jean-François Lyotard, La Condition postmoderne : rapport sur le savoir, Paris, Minuit, 1979.

4. Jean-Pierre Le Goff, La Barbarie douce : la modernisation aveugle des entreprises et de l'école, Paris, La Découverte, 1999.

5. Voir « La wicca, un GN pour les filles? », dans Séverine Abiker, Anne Besson et Florence Plet-Nicolas (dir.), Le Moyen Âge en jeu, Eidôlon, n ${ }^{\circ}$ 86, Pessac, Presses universitaires de Bordeaux, 2009. 
À ce médiévalisme de refuge s'oppose parfois un contre-discours en fantasy qui mérite qu'on s'y intéresse, car il contient une force de dénonciation contre l'idéologie imposée. Un roman comme À la croisée des mondes de Philip Pullman entre ouvertement en lutte contre le sexisme, les idées toutes faites et les religions révélées : c'est de la philosophie politique et de la métaphysique appliquée, le tout disponible en hypermarché - pour tous. En fantasy, on explore des mondes infinis, on entend la voix de la nature et des terres agrestes ou sauvages : ce message, quand Tolkien l'a pour la première fois proféré, était fort révolutionnaire même si on s'y est habitué comme à un poncif depuis. En fantasy, on dénonce le machisme ambiant, on prend la plume quand on est une femme et on y réussit fort bien - voir J. K. Rowling, Robin Hobb, Catherine Dufour, Mélanie Fazi ou Fiona MacIntosh! On se ligue avec la dernière énergie contre tout « mind of metal and wheels ${ }^{6} \gg-$ une tradition, depuis Tolkien. On rêve d'arbres et de campagne, on promeut avec émotion un autre monde possible. Tolkien a de la sympathie pour les Hobbits champêtres, peu nombreux, groupés en quelques petites communes facilement reliées à pied ou à dos de poney, voués à un ordre agraire du monde et farouchement opposés à l'industrie. Les forges de Sauron, celles de Saruman sont porteuses des pires menaces : elles promettent de terrifiants mondes sans issue, voués au totalitarisme carcéral. Tolkien résiste contre son exact contemporain, Big Brother, qui darde son œil noir tandis que le monde agraire passe sous contrôle technologique : Le Seigneur des anneaux et 1984 datent de la même époque, et par des moyens totalement différents, les deux romans atteignent le même but.

La fantasy, héritière de Tolkien aujourd'hui, serait-elle donc une autre forme du cri de Seattle, un cri de résistance contre le monde qu'on nous impose, à la manière du célèbre « un autre monde est possible ${ }^{7} »$ ? La fantasy, avec ses " autres mondes ${ }^{8}$ », serait-elle essentiellement... altermondialiste? En réaction contre un monde qui se représente luimême comme mécanisé, urbain et inhumain, la fiction active l'imaginaire de la petite communauté préservée, des irréductibles Gaulois nantis d'un chaudron magique, bien abrités derrière leurs palissades. Ce n'est plus l'effet Malevil, c'est l'effet Astérix!

À côté de la science-fiction et de la dystopie, la fantasy trace son sillage. Comme les deux autres genres, elle stigmatise le monde actuel et demande

6. J. R. R. Tolkien, The Lord of the Rings, Londres, George Allen and Unwin Ltd, 1954, t. II, The Two Towers, chap. IV, «Treebeard», p. 76.

7. Voir Philippe Pignarre et Isabelle Stengers, La Sorcellerie capitaliste. Pratiques de désenvoûtement, Paris, La Découverte, 2005.

8. Autres mondes, $\mathrm{n}^{\circ} 17$ des Cahiers Robinson, sous la direction d'Anne Besson, mai 2005 (revue du CRELID, Université d'Artois, actes du colloque coorganisé par Anne Besson et Anne-Gaëlle Weber, Université d'Artois, les 28-29 octobre 2004). 
un monde meilleur. Mais tandis que les deux autres genres utilisent l'arme de l'hypotypose - une présenciation fascinée et fascinante du pire - pour mieux accuser, la fantasy parle tout haut de ce qui vit caché et qui s'invente ailleurs que dans les lois du monde actuel : vampires dans la forêt, loupsgarous, femmes, tout ce que le folklore (et non la savante mythologie) a porté en secret pendant des millénaires d'étouffement, tout cela explose au grand jour et entre dans le circuit économique sous la forme de best-sellers suivis de leur sillage de films, jeux, albums et produits dérivés. Le monde médiéval, réputé plus authentique, proche de la nature, des contes et des légendes, cautionne volontiers cette forme de contestation étrangement souple, qui adopte les armes de l'ennemi (mondialisation, matraquage médiatique) pour advenir. En somme, pendant l'ascension résistible du néolibéralisme qui a triomphé dans le monde occidental, la littérature - et non la moindre, du moins en quantité : en première ligne, les best-sellers américains! - a conservé secrètement le précieux souvenir de tout ce qui n'avait pas droit de cité. Les femmes, le folklore, les créatures de la nuit, dont la tradition remonte au XIX ${ }^{\mathrm{e}}$ siècle gothique et fantastique, rappellent l'ambivalence fondatrice des genres «mineurs »: plus la littérature est « petite », « mineure », plus elle est réellement et passionnément lue. Gilles Deleuze aurait dit qu'une ligne minoritaire traverse toujours les majorités les plus arrogantes ${ }^{9}$.

La littérature «médiévalisante » a conservé dans son souterrain (Clarissa Pinkola-Estès parle de $"$ female underworld $\left.{ }^{10} »\right)$ tout ce que le backlash, réaction à la contreculture, s'est alors employé à saccager : le féminisme, l'écologie, ce qu'on n'appelait pas encore altermondialisme, une union entre émotion et politique, une dimension « spirituelle » trop ridiculisée depuis pour qu'on puisse encore employer impunément le mot «spirituel», une remise en cause des religions traditionnelles «à révélation », avec Grand Livre et Grand Homme, le triomphe des autres mondes de l'imaginaire sur « l'autre monde » religieux, la priorité accordée au vivant ici et maintenant et l'humanisme qui consistait à considérer que toute vie avait de la valeur, et qu'aucune valeur extérieure - profit capitaliste ou dictature politique - ne pouvait altérer cet axiome fondamental. Pour résumer, ce qui est en jeu est un courant écologiste, altermondialiste, antipatriarcal et antiraciste.

Ce courant politique «fantasyste » souterrain anti-backlash a pris diverses formes selon les pays. Aux USA, le culte de la déesse-mère dans la wicca, cette néosorcellerie qui est actuellement un «véritable phénomène de société ${ }^{11} »$, entre en écho avec le raz-de-marée des genres

9. François Cusset, La Décennie, op. cit., p. 12.

10. Clarissa Pinkola-Estès, Women who run with wolves. Myths and stories of the Wild Woman Archetype, New York, Ballantine Books, 1992, p. 3.

11. Christian Bouchet, B.A-BA Wicca, Puiseaux, Éditions Pardès, 2000. 
de l'imaginaire, fantasy en première ligne. C'est évident dans les textes de Robin Hobb ou de Philip Pullman, mais même une saga comme Twilight, qui incarne les valeurs religieuses rétrogrades propres à l'Amérique patriarcale (virginité avant le mariage, méditation sur l'âme et Dieu), est porteuse presque malgré elle d'un certain respect de la nature, des femmes, de la tradition folkloriste et de la différence entre les êtres (quand bien même on ne pourrait voir dans cette coexistence de races folkloriques que la représentation fascinée de la différence sexuelle, survalorisée par un état d'adolescence).

Ce qu'on peut dire ainsi de la fantasy, c'est-à-dire des romans seulement, est-il également vrai du medfan, c'est-à-dire du domaine médiéval multisupports dans lequel prend place une certaine fantasy médiévalisante, mais qui l'excède largement? Le medfan est un « univers partagé », une espèce de nébuleuse qui suppose le passage des fictions d'un support à l'autre : livre, $\mathrm{BD}$, série, film, jeu, figurines, produits dérivés, etc. Medfan ne veut pas dire «fan de médiévalisme », comme on pourrait le croire, mais medieval fantastic, ce qui correspond en français à « médiéval merveilleux ». C'est là un vaste domaine, identifiable grâce à une sorte de signalétique immuable. On ne peut pas dire, par exemple que Charmed et Buffy contre les vampires soient spécialement des séries "médiévales », au sens strict; mais ces séries n'en sont pas moins identifiables comme comportant des éléments medfan, à grand renfort de bougies, poignards, grimoires, fumées, créatures monstrueuses, magie, déploiement de force et sorts jetés sur un fond de pavillons ordinaires des suburbs et de décor urbain contemporain.

On mesure donc ce qui sépare la connaissance médiévale historique (reconstitution d'une époque) du medfan, peuplé de créatures de légendes qui n'ont jamais connu l'Histoire. Le medfan tranche sur tout un univers de fidélité médiévale extrême, de reconstitution de bataille, de mariage minutieusement construit dans les moindres détails, de banquet élaboré en déchiffrant des recettes d'époque, de musique jouée sur des instruments authentiques, en bref de dévouée reconstitution du passé ${ }^{12}$. Catégorie graphique et ludico-fictionnelle, le medfan joue grâce à Internet un rôle unificateur et fonctionne comme un signe de ralliement. De site web en site web, il propage donc une signalétique universelle, facilement repérable. Habillage en longues robes seyantes et médiévales, pures aubes druidiques, pratique de rituels, chaudrons, mixtures, bijoux, herbes, pierres précieuses, bougies, tout, dans le Web sorcier médiévalisé, évoque « le côté rose du gothique », si l'on peut dire, en face du dark et du doom.

12. Certains groupes de reconstitution médiévale décernent des brevets de bonne qualité dans le travail de reconstitution (voir le colloque Le Moyen Âge en jeu, op. cit.). 
Il reste à déterminer ce que cette signalétique medfan a de véritablement «médiéval »: on note dans ce type d'illustration des influences manga non négligeables. La cacophonie syncrétique des références n'est pas ressentie comme un problème. Adolescence androgyne, raccourcis saisissants, projection de bras, de pieds ou de poings en premier plan très grossis, postures quelque peu théâtrales et codées, tel est l'apport des mankagas (eux-mêmes fort inspirés de la BD américaine des comics) au medfan international. Quant au décor « médiéval », il est essentiellement... romantique. C'est la peinture romantique européenne ${ }^{13}$, plus particulièrement l'œuvre de Friedrich, des Préraphaélites ou des peintres français auxquels leurs contemporains trouvaient justement un style « troubadour », qui permet d'approcher l'actuel code medfan - de même que, si l'on y réfléchit, le «médiéval» auquel nous faisons naturellement référence est passé par le filtre du Romantisme, de Walter Scott, des contes et légendes et de la cathédrale hugolienne. Tout le talent de Peter Jackson, on le sait, a été de déployer en film des illustrations de Tolkien pour approcher au plus près l'image mentale sommeillant chez la plupart des lecteurs du Seigneur des Anneaux : il a eu recours au talent d'illustrateurs qui avaient formé peu à peu la représentation intérieure qu'on se faisait des lieux et des personnages, ce qui explique que tant de spectateurs du film aient eu le sentiment d'une concordance parfaite avec leur propre vision du livre. Or John Howe et Alan Lee, les illustrateurs en question, ont été formés par l'imagerie romantique ${ }^{14}$. Tour vertigineuse de Saruman, qui surplombe les orages désirés, pics enneigés, nuit et feux, tout cela prend sa source dans la peinture romantique, dont le style medfan est un avatar.

Le style medfan, peut-être en raison de cette prodigieuse puissance signalétique, est d'abord marqué par le triomphe de l'image sur le texte. Au $\mathrm{XIX}^{\mathrm{e}}$ siècle, Alice n'aimait pas les livres sans images, mais les pédagogues se méfiaient, au contraire, d'un médium connoté négativement (regarder les images signifiant qu'on ne savait pas lire, et qu'on ne pouvait donc accéder à la Haute Culture). L'image était le médium du pauvre et de l'ignare, depuis les Bibles en images du Moyen Âge jusqu'aux illustrations gravées des livres romantiques, où la multiplication des dessins accompagne le préjugé de la dégradation populaire du livre. Au Moyen Âge, les peintures et statues servaient aux prêtres à expliquer la Bible au peuple réuni dans l'église : elle était un langage que tout le monde pouvait comprendre, donc un langage facile et simple. Plus tard, l'image a été mutilée : au Xx $\mathrm{Xx}^{\mathrm{e}}$ siècle, elle est photocopiée en noir et blanc faute de moyens, et peu reproduite à cause de dispositions juridiques qui ne frappent pas le texte d'un interdit

13. Excellent panorama dans Le Romantisme, de Jean Clay, Paris, Hachette, 1980.

14. Même si cette imagerie est loin d'être leur seule source d'inspiration. Pour l'anecdote, on peut noter que certaines gravures du Songe de Poliphile annoncent au Xvi siècle les portes de la Moria... 
similaire ${ }^{15}$. Elle a gardé ses connotations de futilité populaire, d'ignorance et de pis-aller culturellement méprisable. Il faudra attendre Internet pour que l'image se déverse à flots sur nos écrans, et pour que sa plasticité lui confère un statut, nouveau pour elle, de médium-roi - mais toujours sans reconnaissance « légitime».

Le Web sorcier est d'abord le Web carnavalesque du renversement effectif de ces valeurs. Le texte recule («nos enfants ne savent plus lire »), l'image triomphe («nos enfants veulent tous faire des écoles d'art»). Parallèlement, le dire-vrai, qui était vécu jusqu'ici comme un impératif moral, s'estompe devant la profusion des légendes urbaines, des avatars, des pseudonymes que choisissent les internautes. L'imaginaire séduit sans qu'on lui reproche son inventivité. Wikipédia, l'encyclopédie en mouvement perpétuel, subit les foudres des tenants de la Haute Culture ${ }^{16}$, mais continue sa route avec allégresse. En somme, le Web est le monde à l'envers : c'est cela qui apparaît comme magique, et «sorcier». Ce n'est pas un hasard si, dans les romans de Catherine Dufour, Internet est le lieu où se sont réfugiées toutes les créatures, dieux, diables, fées ou fantômes qui hantent notre imaginaire ludique et nos souvenirs folkloristes revisités ${ }^{17}$.

C'est surtout parce que le numérique offre une plateforme unique pour décliner, sur des supports faussement divers, des productions homogènes que le néo-médiéval connaît aujourd'hui un tel succès. Grâce à ce tout-numérique, orques et nains glissent du livre au jeu vidéo, du film à la série télévisée, de la photo à l'image animée. Les supports non numériques (livres, bijoux, vêtements, corps humains dans le cas des GN) ne résistent pas à cette emprise qui les stimule : combattre à l'épée semble la chose la plus naturelle du monde, en "vrai», dans la nature, comme sur la bande-annonce du film qu'on visionne sur l'ordinateur. En somme, même les supports non numériques se trouvent, à terme, aspirés par ce système généralisé qui leur offre à la fois leur rendu final et leur diffusion : article sur Internet, fiction sur un blog, dessin au crayon scanné, peinture photographiée, photos prises pendant le GN. Tout finit en numérique.

15. Loi de 1957 : l'unité de texte étant le livre, on peut en citer un fragment; mais l'unité d'image étant, non une œuvre graphique ou plastique, mais chaque image elle-même, il est impossible de citer les images comme on cite les textes.

16. La Révolution Wikipédia, écrit par cinq étudiants de Sciences Po sous la direction de Pierre Assouline, Paris, Mille et Une Nuits, 2007.

17. Catherine Dufour, Blanche-Neige et les lance-missiles, réed. Paris, Le Livre de Poche, 2001. Les spectres du XIX ${ }^{\mathrm{e}}$ siècle (principalement celui du mathématicien Évariste Galois et de Katic, petite bretonne prématurément décédée dont le prénom fait penser à celui de l'auteure) contribuent au développement du télégraphe, puis à celui du réseau ARPANET; enfin, le Web leur offre tout le confort dont ils rêvaient. Ils se réfugient tous dans ce moelleux monde virtuel, propice à l'épanouissement de leur nature spectrale. Katic se modernise et devient alors K@tic. 
La disparition des catégories implantées, la dissolution des valeurs de l'autoproclamée Haute Culture, l'indifférence grandissante du public envers les prétentions de la légitimation littéraire font sans doute, d'un certain point de vue, des ravages ; mais d'un autre point de vue, un certain idéal kantien, jusqu'ici refusé à certaines catégories de livres en raison de leur inscription libraire et éditoriale dans un genre réputé marginal ou populaire, est soudain atteint : si un livre est bon, peu importe son genre, son sous-genre, sa prétendue cible marketing. Il est bon parce qu'un cercle d'admirateurs qui pensent par eux-mêmes l'entourent et le louent. Malgré les prix de la «littérature officielle » qui s'efforcent de braquer leur projecteur sur leur poulain, malgré les écuries des grands faiseurs en matière d'édition, malgré les prétentions inouïes d'une «littérature blanche » lymphatique et de faible niveau, le bon livre est celui que, sans même le secours d'un concept, tous trouvent beau, universellement. C'est rarement celui que les médias et que les éditeurs de littérature blanche voudraient; le Web sorcier est la chance, pour les bons livres de l'imaginaire, de sortir du ghetto. Il "porte», au milieu de tout un fatras de valeur discutable, les vrais livres du monde de demain, qui ne sont pas ceux que Gallimard édite sous une couverture blanche réputée faire autorité. Ce n'est plus le temps, comme jadis les surréalistes, de pourfendre Anatole France ou Claudel : les manières en ont changé; à présent le public vide la place, se détourne poliment, ne prend pas la peine de lutter là contre.

On peut donc se demander en fin de compte si la mouvance «médiévale » n'est pas une forme nouvelle, certes, en ses manières (recours à l'hypnose numérique, prolifération des images suivant une sorte de charte graphique récurrente qui fait office de bannière de ralliement, comme le cri médiéval des rois médiévaux type « Montjoie Saint-Denis! 》) pour un but en réalité bien connu et toujours présent : permettre aux idées de trouver un creuset et de s'écrire dans les livres pour un vrai public. Le medfan fédère un public de «fans » qui n'ont rien contre le livre, lequel joue son rôle au sein d'un « univers partagé » qui l'excède mais le contient. C'est porté par le courant du medfan, son « univers partagé », que le livre de fantasy se signale (au sens de la signalétique) et cible facilement son public. Si nombre de livres de fantasy sont porteurs d'une protestation idéologique contre le système actuel capitaliste, patriarcal et urbain, le medfan, où règne l'image, est tout sauf contestataire. Et c'est pourtant ainsi, par le détour douteux des images et des sites medfan, pourtant non critiques par rapport au monde comme il va, que l'édition de l'imaginaire porte des chefs-d'œuvre non reconnus par les instances de légitimation, mais qui trouvent dans l'engouement vrai du public, comme disait Delacroix de son propre talent de peintre, « jour où se montrer ${ }^{18}$ ».

18. Delacroix, Journal, Paris, Plon, 1981, p. 82 (14 mai 1824). 
Un défaut, souvent souligné par les sociologues, de l'art contemporain est de se faire "sans le public » quand bien même le prétexte de ce public serait répété dans le discours. On peut finir ici avec ce parallèle. L'art contemporain en France (la situation est différente en Angleterre, par exemple), exclut totalement le public, considéré comme une entité imbécile qui « ne comprend pas » et à qui il faut « expliquer » les choses. Ce que le public en réalité ne comprend pas, c'est pourquoi et comment les choses qu'il a sous les yeux sont arrivées dans le musée; elles ne suscitent en effet aucune adhésion collective, et personne ne se rassemble autour d'elles de manière universelle quoique sans concept, pour reprendre une fois de plus la formulation kantienne. Mais les promoteurs de l'art contemporain n'ont que le souci de faire monter la cote des «artistes». Une « écurie » comme la galerie Emmanuel Perrotin (qui représente entre autres Jeff Koons, Xavier Veilhan, Murakami) joue un rôle de premier plan dans la programmation de l'art contemporain à Versailles (qui a présenté et présentera des expositions de Jeff Koons, Xavier Veilhan, Murakami). Les liens entre ces poulains et François Pinault, spéculateur du monde de l'art qui a financé pour une grande part les expositions versaillaises, montre assez comment le fameux " public », à qui prétendument seraient destinées ces manigances, est réduit à un pur prétexte, une « couverture ». On peut se demander si ce processus, évident en art contemporain, n'aurait pas son pendant en littérature blanche : si le monde de l'art contemporain est naturellement sans public, car c'est une monnaie (or qui aura à cœur de contempler un billet de banque?) le monde officiel du livre serait-il sans lecteurs?

Il est difficile d'en savoir plus car le public, docile, se détourne poliment, sans protester, laissant courir les discours mensongers sur l'art et la littérature, sans chercher à lutter contre eux. On retrouve la même attitude générale qu'en littérature blanche : on délaisse au lieu de combattre, on se détourne au lieu d'affronter le mensonge, on se réfugie sur la Toile au lieu de protester dans le monde extérieur. L'art en tant qu'oligopole, devenu monnaie, et la littérature blanche prétendent ainsi se passer du peuple, un peuple qui se rétracte et se réfugie ailleurs, dans la fiction, dans un monde meilleur mais peut-être fallacieux. La scène officielle est peuplée de fantoches, tandis que les vrais lecteurs, les vrais spectateurs, le vrai art et la vraie littérature sont résolument ailleurs.

La question «peut-on se passer du peuple? » sonne inquiétante dans le domaine politique, et à peine moins dans celui de l'art et de la littérature. Pourtant, les formes symboliques que sont l'art et la littérature ont plus que jamais leur rôle à jouer en politique, et le médiévalisme en fournit quelques exemples. C'est en référence à la fiction tolkienienne qu'on clamait dans les manifestations des slogans comme « Frodo est vivant» ou « Gandalf président! », sur la côte californienne dans les années de la contre-culture. Le pouvoir de la fiction n'est pas seulement utile pour créer de modernes 
mythologies contre les discours politiques ambiants : on peut en effet se demander si la nouvelle forme de protestation indirecte qu'est aujourd'hui l'abandon du terrain de la lutte, la désaffection, le refuge dans la fiction qui détourne apparemment du « réel », ne devrait pas être examinée avant d'être jugée comme irrecevable et antipolitique, car elle contient peutêtre un potentiel de résistance élastique plus fort qu'on le croit : elle met en œuvre une sorte de résistance souple, peut-être une non-violence à la Gandhi. Cela reste en tout cas à interroger.

Anne Larue

Université Paris 13-Paris Nord (CENEL) 